\title{
Ultrastructural study of the human cerebellar cortex
}

\begin{abstract}
We analyzed the fine structure and the synaptical formations in the cerebellar cortex of ten unimpaired human subjects by the use of electron microscopy Such ultrastructural studies of the human cerebellum, especially of the areas where our cytoarchitectonic knowledge is still incomplete, will provide a better understanding of the nonmotor contribution of the cerebellum in cognition and behavior and also its role in neurodegenerative and demyelinating diseases such as Alzheimer's and multiple sclerosis.
\end{abstract}

Keywords: cerebellar cortex, Purkinje cells, granular cells, molecular layer, climbing fibers, mossy fibers
Volume 9 Issue 2 - 2019

\author{
Euphrosyni Koutsouraki, Dimitrios \\ Michmizos, Vassiliki Costa, Stavros Baloyannis \\ Aristotle University, School of Medicine, Thessaloniki, Greece
}

\begin{abstract}
Correspondence: Euphrosyni Koutsouraki MD, PhD, Ass. Professor of Neurology-Neuroimmunology, Aristotle University, A' Neurologic clinic, AHEPA Hospital, St. Kyriakidi I, 54636, Thessaloniki, Greece, Tel 00302310 994604, Fax 00302310 9947। I,Email efrosink@gmail.com
\end{abstract}

Received: January 13, 2019 | Published: March 13, 2019

\section{Introduction}

Apart from its appearance in numerous imaging studies, the human cerebellum has received little attention in the recent past years. ${ }^{1}$ The human cerebellum is ample, in particular the great width of the folia of the hemispheres which is one of its distinguishing features. A faint depression on the superior surface marks the border between the vermis and the hemispheres; Schmahmann et al. ${ }^{2}$ were probably right when they concluded that this border is quite indistinct. This superior surface of the cerebellum consists of the anterior lobe and Bolk's lobulus simplex. Bolk coined this term because of the lack of any clear border between the vermis and the hemispheres results this lobule having a uniform appearance.

In both the anterior lobe and the lobulus simplex, the transverse fissures run uninterruptedly from the vermis into the hemispheres. Thus, the anterior lobe and the lobulus simplex constitute a single morphological entity. ${ }^{3}$ The entire cerebellum is canopied by a uniformly structured cortex. Due to the transverse fissures, the length of the cortical sheet greatly exceeds its width. The cortex is continuous between the successive segments (lobules) of the folial chains of the vermis and the hemispheres, with the exception of the cortex of the flocculus, which is not continuous with the tonsilla. Between the caudal vermis and the hemispheres the cortex is usually interrupted.

The cerebellar cortex has three layers: the molecular layer, the layer of Purkinje cells and the granular layer and contains five types of neurons: the granular neurons, Golgi type II neurons, stellate neurons, basket cells and the Purkinje cells. ${ }^{4,5}$ There are two types of afferent fibers, the climbing fibers and the mossy fibers. The climbing fibers originate from the inferior olivary nucleus and the anatomical ratio between former and the Purkinje cells is one-to-one. ${ }^{6}$ The mossy fibers are the main afferent pathway to the cerebellum and originate from all the other parts of the CNS with the exception of the inferior olivary nucleus. ${ }^{7}$

The mossy fibers terminate in rosette-like terminals, which develop multisynaptic contacts with granule cells and Golgi cells, called glomerulus. ${ }^{8}$ A glomerulus consists of: (1) Rosette-like endings of mossy fibers, (2) Claw-like dendritic terminals of the granule cells, (3) Dendritic branches and axons of Golgi type II neurons; thus, the mossy fibers make synaptic contact with both axons and dendrites of
Golgi II neurons, and (4) Glial sheaths which surround each of the glomerulus

Both climbing and mossy fibers produce excitatory synapses using acetylcholine and glutamic acid. We could not manage to find any significant morphological differences between cholinergic and glutaminergic synaptic vesicles. The only way to differentiate the two kinds of neurotransmitters is by using monoclonal antibodies.

There is also another afferent pathway, the axons of the granular cells, the so called parallel fibers, which terminate on the dendrites of Purkinje cells. ${ }^{9}$ Both the climbing and parallel fibers use acetylcholine, glutamic acid and neuropeptides producing excitatory synapses. Inside the endings of the climbing fibers we can also see dopaminergic vesicles, identified by their osmiophilic center. In order to further explore the unltrastructure of the cerebellar formation, we analyzed the ultrafine structures and the synaptic formations in the cerebellar cortex of ten unimpaired human cerebella under electron microscopy.

\section{Materials and methods}

Brain tissue was obtained at autopsy from ten intact brains, derived from healthy individuals who died accidentally, six men and four women, aged 30-50 years. After death, the autopsy was performed within $6 \mathrm{~h}$. The brains were excised at a temperature of 4 ${ }^{\circ} \mathrm{C}$. Multiple samples from the cerebellum were taken and immersed in Sotelo's fixing solution, consisted of $1 \%$ paraformaldehyde, $2.5 \%$ glutaraldehyde in cacodylate buffer $0.1 \mathrm{M}$, adjusted at $\mathrm{pH}$ 7.35 , where they remained for $3 \mathrm{~h}$ at $4{ }^{\circ} \mathrm{C}$. They were postfixed in $1 \%$ osmiumtetroxide for $30 \mathrm{~min}$ and dehydrated in graded alcohol solutions and propylene oxide. They were embedded in araldite mixture. Thin sections at silver interference color were performed in a Reichert ultratome and contrasted with uranyl acetate and lead citrate. The grids were studied in a Zeiss $9 \mathrm{aS}$ electron microscope.

\section{Results and discussion}

There are four kinds of inhibitory neurons in the cerebellar cortex. The basket cells, the stellate neurons and the Golgi II neurons that produce inhibitory synapses with Purkinje cells using mainly GABA. GABAenergic vesicles are ellipsoid in contrast to the cholinergic which are spheroid. This why Gray named the cholinergic, vesicles S 
whereas the GABAenergic, vesicles E. The fourth kind of inhibitory neurons are the Purkinje cells which inhibit the cells of the dentate nucleus. We must mention that both basket cells and stellate cells become excited by the parallel fibers whereas the Golgi II cells by the mossy (Figure 1).

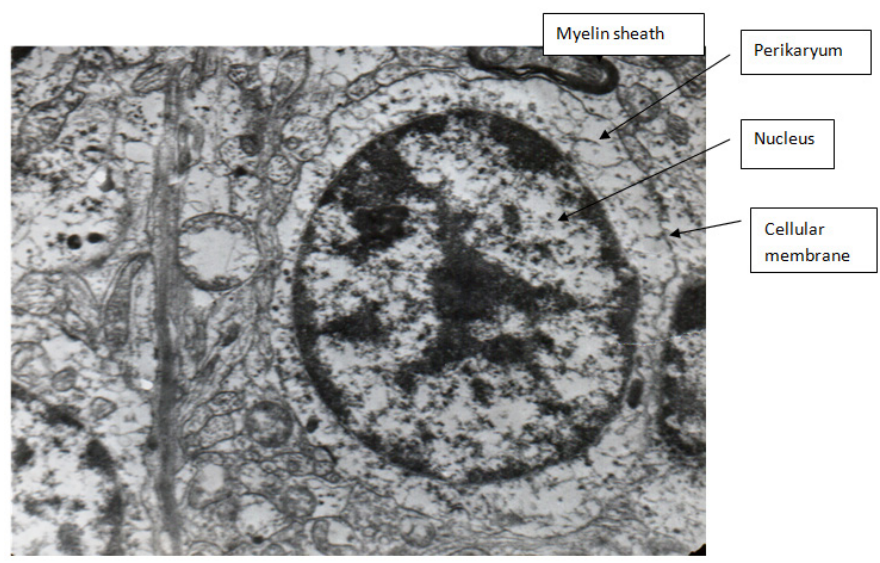

Figure I Stellate cell from the molecular layer of the human cerebellum $(48.400 x)$

The granular layer consists of the granular cells, the Golgi type II neurons, the microglial cells of Hortega, the terminals of the mossy fibers, the myelinated recurrent collaterals of Purkinje cells and the astroglial sheaths (Figure 2). The granular cells are characterized by a big nucleus which contains heterochromatin attached to the nuclear membrane, nucleolus, euchromatin, smooth endoplasmatic reticulum, small spheroid mitochondria and (mainly) unattached ribosomes. The axons of the granular cells are divided, forming the parallel fibers which are contacted by the dendrites of the Purkinje cells. The microglial cells of Hortega consist of a dark oblong nucleus and dark perikaryum with small mitochondria, many free ribosomes and cisternae of the smooth endoplasmatic reticulum. The Golgi type II neuron is four or five times as big as the granular cell. It consists of a big nucleus (containing mainly euchromatin), nucleolus, small mitochondria, smooth endoplasmatic reticulum, Golgi apparatus, many free ribosomes and lysosomes. Sometimes the endoplasmatic reticulum, the Golgi apparatus and the lysososmes are contacted, producing a formation which was called GERL. ${ }^{10}$ Cerebellar cortex has its own neuroglia, the neuroepithelial Golgi cell, which makes astroglial sheaths round the dendrites of Purkinje cells. When the Purkinje cells degenerate the neuroepithelial cells become hyperplastic and form the neuroglia of Bergmann. This neuroglia is the evidence of a chronic cerebellar degeneration.

The characteristic neuron of the cerebellar cortex is the Purkinje cell. Its nucleus is quite big and contains a nucleolus which is surrounded by fibrils of euchromatin. The entire nucleus is surrounded by many cisternae from the Golgi apparatus which develops contacts with many spheroid vesicles. Most of the ribosomes are attached to the cisternae of the endoplasmatic reticulum forming the granular or rough endoplasmatic reticulum (Figure 3). There is also a large number of mitochondria which demonstrate multiplicity and diversity of shape and form. The lysosomes are characterized as type I (small with no content), type II (bigger and with osmiophilic content) and the huge lysosomes with lipid content which are to be found mainly in elderly people.

The most impressing element of a Purkinje cell is its dendritic network. There is a top dendrite which is divided into secondary, tertiary, quaternary and so on branches. On the surface of these dendrites are dendritic spines, the main postsynaptic elements of the cerebellar cortex. Each of these spines contains a mitochondrion, many elongated cisternae forming the apparatus of the spine, numerous fibrils and cisternae of the smooth endoplasmatic reticulum. We must also mention the presence of many unattached spines which do not demonstrate any postsynaptic differentiation, more numerous in the younger individuals, providing the substrate for the neuronal plasticity of the cerebellar cortex.

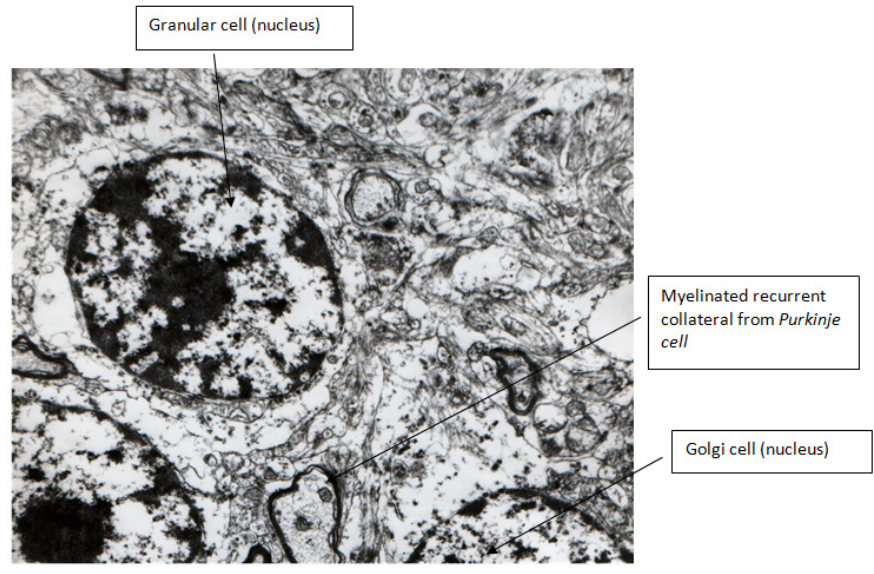

Figure 2 Granular cells, a Golgi II cell and myelinated recurrent collaterals from Purkinje cells from the granular layer of the human cerebellum (24.400x).

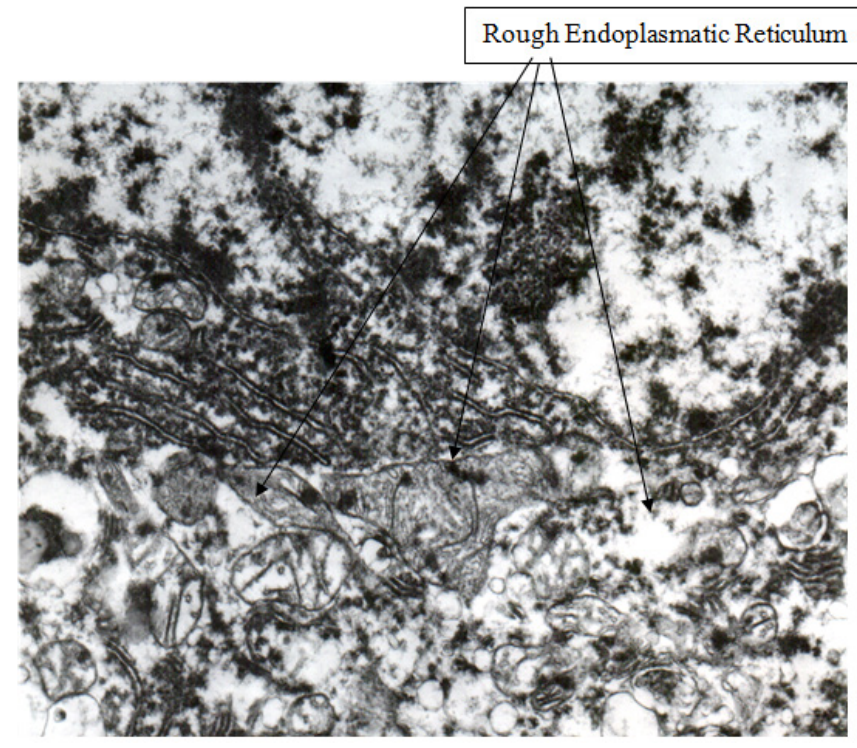

Figure 3 Large number of ribosomes attached to the cisternae of the rough endoplasmatic reticulum in the perikaryum of a Purkinje cell of human cerebellum (48.800x).

In conclusion, ultrastructular studies of the human cerebellum, especially studies of the areas for which our cytoanatomical knowledge is still limited, such as the present one, can provide with a better understanding of the non-motor contribution of the cerebellum in cognition and behavior as well as its role in neurodegenerative and demyelinating diseases such as Alzheimer's disease and multiple sclerosis.

\section{Acknowledgments}

None. 


\section{Conflicts of interest}

Authors declare no conflicts of interest.

\section{References}

1. Voogd J. The human cerebellum. J Chem Neuroanat. 2003;26(4):243252.

2. Schmahmann JD, Doyon J, McDonald D, et al. Three dimensional MRI atlas of the human cerebellum in proportional stereotaxic space. Neuroimage. 1999;10(3 pt 1):233-260.

3. Bolk L. Das Cerebellum der Saugetiere. Eine vergleichend anatomische Untersuchung. F. Bohn. Fischer, Jena; 1906.

4. Koutsouraki E. The neurotransmitters of the mossy fibres of the human cerebellum. Thesis. Aristotle University, Thessaloniki, Greece; 1996.

5. Koutsouraki E, Anastasiades J, Baloyannis S. An Immunohistochemical Study of N-Methyl-D-Aspartate Receptors in Human Cerebellum and Hippocampus. Am J Med Sci Med. 2013;1(2):28-30.
6. Baloyannis S. Pathological alterations of the climbing fibres of the cerebellum in vascular dementia: A Golgi and electron microscope study. J Neurol Sci. 2007;257(1-2):56-61.

7. Delvendahl I, Weyhersmüller A, Ritzau-Jost A, et al. Hippocampal and cerebellar mossy fibre boutons - same name, different function. $J$ Physiol. 2013;591(13):317-3188.

8. Hámori J, Szentágothai J. Participation of Golgi neuron processes in the cerebellar glomeruli: An electron microscope study. Exp Brain Res. 1966;2(1):35-48.

9. Ekerot CF, Jömtell H. Parallel fiber receptive fields: a key to understanding cerebellar operation and learning. Cerebellum. 2003;2(2):101-109.

10. Novikoff AB. GERL, its form and function in neurons of rat spinal ganglia. Biol Bull (Woods Hole). 1964;127:358. 